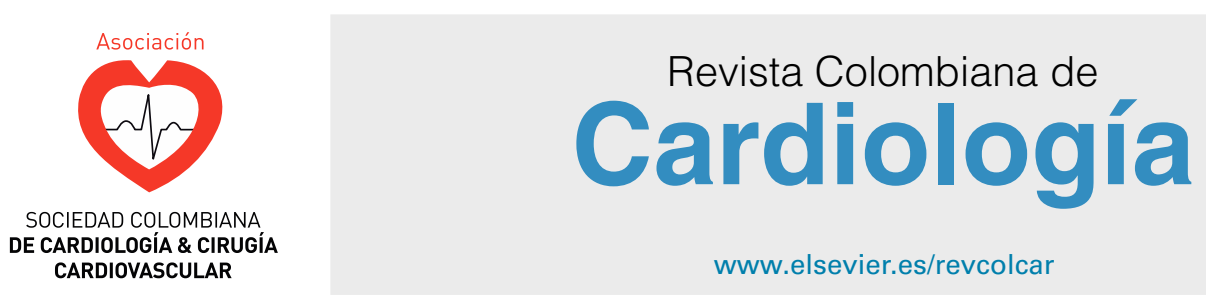

\title{
EDITORIAL
}

\section{Visión de la insuficiencia cardiaca avanzada. ¿Es demasiado tarde?}

\section{A view of advanced heart failure. Is it too late?}

\author{
Clara Inés Saldarriaga Giraldo ${ }^{\mathrm{a}, \mathrm{b}, \mathrm{c}}$ \\ a Programa de insuficiencia cardiaca, Clínica CardioVID. Medellín, Colombia \\ b Programa de Cardiología, Universidad Pontificia Bolivariana, Medellín, Colombia \\ c Universidad de Antioquia, Medellín, Colombia
}

Recibido el 23 de agosto de 2019; aceptado el 26 de agosto de 2019

Detectar el punto de no retorno en muchas de las enfermedades médicas a las que se enfrenta el clínico en su práctica diaria, es un "arte" que se desarrolla a partir de la experiencia y la frecuencia con la que está expuesto a tratar pacientes con una dolencia en particular. En muchas oportunidades, cuando se tiene en frente a un paciente con insuficiencia cardiaca avanzada que finalmente fue referido a un programa multidisciplinario, después de haber pasado por múltiples hospitalizaciones y cuando su calidad de vida y su pronóstico están seriamente comprometidos, surgen varias preguntas:

\section{¿Qué se hizo mal?}

¿Se falló en la prevención de la enfermedad?, ¿no se controlaron de manera adecuada los factores de riesgo cardiovasculares en la población?, ¿no se diagnosticó a tiempo esta enfermedad?, ¿no hubo preocupación por encontrar la causa de la alteración de la función ventricular para

Correo electrónico: clarais@une.net.co tratar de corregirla y con frecuencia no se ofreció de manera oportuna a los pacientes toda la terapia farmacológica y no farmacológica que ha mostrado modificar el curso clínico de la enfermedad?

\section{¿Es demasiado tarde?}

Probablemente falló la capacidad de detectar el peligro. Por fortuna, la acepción de esa "delgada línea roja" que señala la presencia de un pronóstico desfavorable, se ha simplificado recientemente gracias a la publicación de consensos que actualizan la definición de insuficiencia cardiaca avanzada e involucran factores más allá de la fracción de eyección y la clase funcional, como hospitalizaciones recurrentes, uso de inotrópicos, caquexia cardiaca, choques frecuentes del desfibrilador, intolerancia al tratamiento farmacológico, requerimientos de altas dosis de diurético para controlar edemas, compromiso de otros órganos como riñón e hígado y disminución severa de la capacidad física en mediciones objetivas como la prueba cardiopulmonar o la caminata de seis minutos. Estas características pueden aparecer, incluso, en pacientes con insuficiencia cardiaca con 
función preservada o que se encuentran en clase funcional II de la clasificación de la Asociación del Corazón de Nueva York (NYHA, su sigla en inglés por New York Heart Association) $)^{1,2}$.

Es importante recordar que los pacientes con insuficiencia cardiaca aguda también pueden tener criterios de enfermedad avanzada y necesitar intervenciones como soporte ventricular temprano. Por esta razón, se han implementado exitosamente en el mundo estrategias como el código choque y la aplicación de la clasificación INTERMACS $^{3}$, que permiten tomar decisiones tempranas que evitan el deterioro irreversible de otros órganos y sistemas.

\section{¿Qué tratamiento faltó considerar?}

Las alternativas de tratamiento dependen de la edad, el pronóstico y las comorbilidades ${ }^{4}$. El trasplante de corazón es la opción de tratamiento para los pacientes con insuficiencia cardiaca avanzada que tienen indicación para este; sin embargo, es un recurso limitado por la disminución del número de donantes y el aumento de pacientes en lista de espera y el principal problema es la referencia de pacientes de manera tardía, cuando el compromiso severo de múltiples órganos e hipertensión pulmonar irreversible contraindican esta opción. La asistencia ventricular es una realidad en Colombia, pero existen limitaciones de costos y disponibilidad limitada, y pese a que los cuidados paliativos son una excelente opción cuando no existen contraindicaciones para la terapia avanzada, hay gran desconocimiento de esta alternativa y se la tiene en cuenta de manera tardía, a sabiendas de su efectividad para mejorar la calidad de vida del paciente y su familia ${ }^{5,6}$.

Conviene recordar que el tratamiento actual de la enfermedad ha mejorado la expectativa de vida y por esta razón se ha incrementado el número de pacientes que llegan a la fase avanzada. Esta población representa solo el 1\% del total; sin embargo, por su alta frecuencia de hospitalizaciones recurrentes genera un consumo importante de recursos que explican el $60 \%$ de los costos de atención 7 .

Como sociedad y como sistema de salud se tienen grandes retos para impedir que los pacientes con insuficiencia cardiaca continúen llegando "demasiado tarde". El principal es realizar educación médica para mejorar la capacidad de identificar oportunamente a esta población y permitir su remisión. Esto solo será posible si se vence la fragmentación en la atención en salud y se logra eficiencia en la implementación de una ruta integrada que permita la comunicación entre los médicos desde la atención primaria, hasta la atención especializada ${ }^{8}$. También es prioritario capacitar al personal médico para identificar los pacientes que se benefician de cuidados paliativos, mejorando su capacidad de discutir la necesidad de cambiar los objetivos de tratamiento, y optimizando las habilidades de comunicación y la relación con el paciente y su familia para abordar el tema de las decisiones al final de la vida.

Ninguna de estas intervenciones será posible si no se cuenta con el compromiso de las aseguradoras para garantizar el acceso de los pacientes a un seguimiento multidisciplinario en programas especializados de insuficiencia cardiaca. Esta es la mejor manera de tomar decisiones acertadas sobre cuál es la mejor alternativa terapéutica: siendo racionales en la utilidad y la indicación de las intervenciones en el "momento justo"'.

\section{Bibliografía}

1. Fang JC, Ewald GA, Allen LA, Butler J, Westlake Canary CA, Colvin-Adams M, et al. Advanced (stage D) heart failure: a statement from the Heart Failure Society of America Guidelines Committee. J Card Fail. 2015;21:519-34.

2. Crespo-Leiro MG, Metra M, Lund LH, Milicic D, Costanzo MR, Filippatos G, et al. Advanced heart failure: a position statement of the Heart Failure Association of the European Society of Cardiology. Eur J Heart Fail. 2018;20:1505-35.

3. Stevenson LW, Pagani FD, Young JB, Jessup M, Miller L, Kormos RL, Naftel DC, Ulisney K, Desvigne-Nickens P, Kirklin JK. INTERMACS profiles of advanced heart failure: the current picture. J Heart Lung Transplant. 2009;28:535-41.

4. Ponikowski P, Voors AA, Anker SD, Bueno H, Cleland JG, Coats AJ, et al. 2016 ESC Guidelines for the diagnosis and treatment of acute and chronic heart failure: The Task Force for the diagnosis and treatment of acute and chronic heart failure of the European Society of Cardiology (ESC). Developed with the special contribution of the Heart Failure Association (HFA) of the ESC. Eur J Heart Fail. 2016;18:891-975.

5. Rogers JG, Patel CB, Mentz RJ, Granger BB, Steinhauser KE, Fiuzat $M$, et al. Palliative care in heart failure: the PAL-HF randomized, controlled clinical trial. J Am Coll Cardiol. 2017;70:331-41.

6. O’Donnell AE, Schaefer KG, Stevenson LW, DeVoe K, Walsh K, Mehra MR, Desai AS. Social Worker-Aided Palliative Care Intervention in High-risk Patients With Heart Failure (SWAP-HF): a pilot randomized clinical trial. JAMA Cardiol. 2018;3:516-9.

7. Frieder Braunschweig, Martin R, Cowie, Angelo, Auricchio. What are the costs of heart failure? EP Europace. 2011;13(S2):ii13-7.

8. Torres A, Gómez E. Unidades de falla cardiaca: una propuesta para el sistema de salud colombiano. Rev Col Cardiol. 2016;23(S1):13-9.

9. Saldarriaga C, Garcés J, Agudelo A, Guarín L, Mejía J. Impacto clínico del programa de la falla cardiaca en un centro de referencia cardiovascular. Revista Colombiana de Cardiología. 2016;23:260-326. 